\title{
Application Research of the Sparse Representation of Eigenvector on the PD Positioning in the Transformer Oil
}

\author{
Qing Xie, ${ }^{1,2}$ Dan Liu, ${ }^{2}$ Ying Zhang, ${ }^{2}$ Shuguo Gao, ${ }^{3}$ Tong Li, \\ Xinjie Wang, ${ }^{4}$ and Fangcheng $L \ddot{u ̈}^{2}$ \\ ${ }^{1}$ State Key Laboratory of Alternate Electrical Power System with Renewable Energy Sources, \\ North China Electric Power University, Baoding, Hebei 071003, China \\ ${ }^{2}$ Hebei Provincial Key Laboratory of Power Transmission Equipment Security Defense, \\ North China Electric Power University, Baoding, Hebei 071003, China \\ ${ }^{3}$ State Grid Hebei Electric Power Research Institute, Shijiazhuang 050021, China \\ ${ }^{4}$ Department of English, North China Electric Power University, Baoding, Hebei 071003, China \\ Correspondence should be addressed to Qing Xie; xq_ncepu@126.com
}

Received 8 April 2016; Revised 23 August 2016; Accepted 27 September 2016

Academic Editor: Elias Aboutanios

Copyright (C) 2016 Qing Xie et al. This is an open access article distributed under the Creative Commons Attribution License, which permits unrestricted use, distribution, and reproduction in any medium, provided the original work is properly cited.

\begin{abstract}
The partial discharge (PD) detection of electrical equipment is important for the safe operation of power system. The ultrasonic signal generated by the PD in the oil is a broadband signal. However, most methods of the array signal processing are used for the narrowband signal at present, and the effect of some methods for processing wideband signals is not satisfactory. Therefore, it is necessary to find new broadband signal processing methods to improve detection ability of the PD source. In this paper, the direction of arrival (DOA) estimation method based on sparse representation of eigenvector is proposed, and this method can further reduce the noise interference. Moreover, the simulation results show that this direction finding method is feasible for broadband signal and thus improve the following positioning accuracy of the three-array localization method. And experimental results verify that the direction finding method based on sparse representation of eigenvector is feasible for the ultrasonic array, which can achieve accurate estimation of direction of arrival and improve the following positioning accuracy. This can provide important guidance information for the equipment maintenance in the practical application.
\end{abstract}

\section{Introduction}

Electrical equipment working status is directly related to the reliable operation of power system. And the practice has proved that the $\mathrm{PD}$ is the main reason for the high voltage electrical equipment insulation breakdown finally. In order to avoid accidents and timely find the potential danger, it is necessary for the electrical equipment partial discharge testing to ensure system reliability [1-6].

In the PD detection, an array sensor is used to collect ultrasonic signals generated by the PD. Then the array signal processing technology is used to complete the source direction of arrival (DOA) estimation and positioning. This method not only has strong anti-interference ability, but also has high positioning accuracy, and it has been widely used in many areas [7-9]. However, the main processing object of the traditional array signal processing methods is a narrowband signal, and the corresponding variety of space spectrum estimation (direction of arrival, DOA) methods that have high resolution and fast computing speed have been successfully applied. The electrical equipment ultrasonic signal generated by the PD in transformer oil is a typical broadband signal $[10,11]$, so the study on the DOA estimation algorithm that is suitable for wideband signal has extremely important significance.

The more classical wideband direction finding algorithm is mainly divided into two categories.

The first kind of method is incoherent subspace algorithm (ISM algorithm) [12, 13]. It is that a broadband signal is divided into a number of narrowband signals, and the average value is obtained after estimating the DOA of each narrowband signal. This method is a simple average of the 
narrowband signal processing results, which has a large amount of calculation, and cannot overcome the shortcomings of subspace algorithms adopted by the narrowband signal, such that it is easily affected by noise and sampling points and cannot solve the coherent sources. The second method is the coherent signal subspace algorithm (CSM algorithm) [14-17]. A focusing matrix is used to focus on all frequency components on a single reference frequency. Narrowband signal processing method is used to estimate the DOA of the covariance matrix after focusing, which reduces the correlation coefficient between signals, and can achieve the goal of coherent solution. Moreover, the existing CSM algorithm has to use the traditional narrowband signal processing method after focusing, which is still unable to avoid the disadvantages of subspace algorithms.

Mallat and Zhang in 1990s proposed the theory of signal sparse decomposition $[18,19]$. It can be constructed by using different ways according to the specific signal form and different research purposes. Although, the signal is represented by a handful of basis functions, the information in the signal also focuses on these few basis functions, so it is more conducive to extract and explain the essential characteristics of signals. At present, the signal sparse decomposition has been widely used in signal noise reduction, compression, coding and image processing, and other fields [11]. In this paper, the sparse decomposition theory is applied to the PD signal DOA estimation. According to the array signal direction vectors, an overcomplete atom dictionary is established. The matching pursuit (MP) algorithm is used to choose the appropriate atoms, and the angle information contained in the atoms is the DOA of signal sources.

This work studies the PD positioning method in the transformer oil based on the sparse representation of eigenvectors. Taking a nine-element circular ultrasonic array sensor as an example, the mathematical model of ultrasonic array signals is given. Firstly, the broadband PD signals are received by an ultrasonic array sensor, and the covariance matrix of a single frequency is obtained by using RSS focusing method [20]. Then an eigenvector corresponding to the maximum eigenvalue is obtained through eigendecomposition of the covariance matrix obtained; the eigenvector is as the amount to be decomposed. According to the reference frequency and the steering vector form of an array signal, a step and step overcomplete dictionary is established, and thus the DOA estimation of the PD signal can be obtained by MP. Moreover, this method can further reduce the noise interference. Finally, according to the results, the PD source is located by using the three-array cross positioning principle. The simulation and experimental results show that the direction finding method based on sparse representation of eigenvectors can get higher accuracy of the DOA estimation results and improve the subsequent positioning precision.

\section{Broadband PD Ultrasonic Array Signal}

2.1. The Mathematical Model of Array Signal. The research results show that the ultrasonic frequency produced by the $\mathrm{PD}$ in transformer oil is mainly concentrated in the range of
$50 \mathrm{kHz}$ to $400 \mathrm{kHz}$, the center frequency is between $70 \mathrm{kHz}$ and $200 \mathrm{kHz}$, and so the PD ultrasonic signal source is a typical broadband signal.

Assuming that a uniform array consists of $M$ equally spaced elements and there is a space with $P$ broadband signals, the incident angle is, respectively, $\varphi_{1}, \varphi_{2}, \ldots, \varphi_{P}$, and the signal received from the $K$ th element can be expressed as

$$
x_{k}(t)=\sum_{i=1}^{P} s_{i}\left[t-\tau_{k}\left(\varphi_{i}\right)\right]+n_{k}(t),
$$

where $s_{i}(t)(i=1,2, \ldots, P)$ is incident broadband signal; $n_{k}(t)$ is additive noise; $\tau_{k}\left(\varphi_{i}\right)$ is time difference relative to the reference node when the $i$ th signal source is received by the $k$ th element.

The time shift theorem of Fourier transform is as follows: a signal is carried on Fourier transform after the signal has a time shift equal to that of the signal that has a phase delay after Fourier transform. If $s(f)$ is the Fourier transform form of $s(t)$, that is,

$$
\operatorname{FFT}[s(t)]=s(f),
$$

then the Fourier transform form of $s(t+\tau)$ is

$$
\operatorname{FFT}[s(t-\tau)]=s(f) e^{-j 2 \pi f \tau} .
$$

For the signal received by the $k$ th element, both sides of (1) are analyzed based on Fourier transform:

$$
x_{k}(f)=\sum_{i=1}^{P} s_{i}(f) e^{-j 2 \pi f \tau_{k}\left(\varphi_{i}\right)}+n_{k}(f) .
$$

The Fourier transform for $M$ elements can be written in matrix form, which is

$$
\begin{aligned}
& {\left[\begin{array}{c}
x_{1}(f) \\
x_{2}(f) \\
\vdots \\
x_{M}(f)
\end{array}\right]} \\
& =\left[\begin{array}{cccc}
e^{-j \omega \tau_{1}\left(\varphi_{1}\right)} & e^{-j \omega \tau_{1}\left(\varphi_{2}\right)} & \ldots & e^{-j \omega \tau_{1}\left(\varphi_{P}\right)} \\
e^{-j \omega \tau_{2}\left(\varphi_{1}\right)} & e^{-j \omega \tau_{2}\left(\varphi_{2}\right)} & \ldots & e^{-j \omega \tau_{2}\left(\varphi_{P}\right)} \\
\vdots & & \ddots & \vdots \\
e^{-j \omega \tau_{M}\left(\varphi_{1}\right)} & e^{-j \omega \tau_{M}\left(\varphi_{2}\right)} & \ldots & e^{-j \omega \tau_{M}\left(\varphi_{P}\right)}
\end{array}\right]\left[\begin{array}{c}
s_{1}(f) \\
s_{2}(f) \\
\vdots \\
s_{P}(f)
\end{array}\right] \\
& +\left[\begin{array}{c}
n_{1}(f) \\
n_{2}(f) \\
\vdots \\
n_{M}(f)
\end{array}\right] .
\end{aligned}
$$

And they can be written as

$$
\mathbf{X}(f)=\mathbf{A}(f, \theta) \mathbf{S}(f)+\mathbf{N}(f) .
$$


Among them, the steering vector matrix is

$$
A(f, \varphi)=\left[\begin{array}{cccc}
e^{-j \omega \tau_{1}\left(\varphi_{1}\right)} & e^{-j \omega \tau_{1}\left(\varphi_{2}\right)} & \cdots & e^{-j \omega \tau_{1}\left(\varphi_{P}\right)} \\
e^{-j \omega \tau_{2}\left(\varphi_{1}\right)} & e^{-j \omega \tau_{2}\left(\varphi_{2}\right)} & \cdots & e^{-j \omega \tau_{2}\left(\varphi_{P}\right)} \\
\vdots & \vdots & \ddots & \vdots \\
e^{-j \omega \tau_{M}\left(\varphi_{1}\right)} & e^{-j \omega \tau_{M}\left(\varphi_{2}\right)} & \cdots & e^{-j \omega \tau_{M}\left(\varphi_{P}\right)}
\end{array}\right]
$$

The signal direction matrix $A(f, \varphi)$ is different from narrowband direction matrix. Here, the frequency is the whole band of the signal, while the frequency is a single fixed value in a narrowband model.

When the signal is analyzed based on the discrete Fourier transform (DFT) with $J$ points, the frequencies are $J$ discrete points, and then (6) can be discrete as

$$
X\left(f_{j}\right)=A\left(f_{j}\right) S\left(f_{j}\right)+N\left(f_{j}\right) \quad j=1,2, \ldots, J .
$$

The steering vector matrix is

$$
A\left(f_{j}, \varphi\right)=\left[a\left(\varphi_{1}, f_{j}\right) a\left(\varphi_{2}, f_{j}\right) \cdots a\left(\varphi_{P}, f_{j}\right)\right]
$$

where $\mathbf{a}\left(\varphi_{i}, f_{i}\right) \quad(i=1,2, \ldots, k)$ is a steering vector:

$$
\mathbf{a}\left(\varphi_{\mathbf{i}}, \mathbf{f}_{\mathbf{j}}\right)=\left[\begin{array}{c}
\mathrm{e}^{-\mathbf{j} 2 \pi \mathbf{f}_{\mathbf{j}} \tau_{1}\left(\varphi_{\mathbf{i}}\right)} \\
\mathrm{e}^{-\mathbf{j} 2 \pi \mathbf{f}_{\mathbf{j}} \tau_{2}\left(\varphi_{\mathbf{i}}\right)} \\
\vdots \\
\mathrm{e}^{-\mathbf{j} 2 \pi \mathbf{f}_{\mathbf{j}} \tau_{\mathbf{M}}\left(\varphi_{\mathrm{i}}\right)}
\end{array}\right] .
$$

2.2. The Structure of the Circular Ultrasonic Array Sensor. The circular ultrasonic array sensor is composed of $M$ identical elements evenly distributed on the circumference with a radius of $R$ in the $x-y$ plane; the elements are arranged as shown in Figure 1 (e.g., taking nine element). The coordinate system of the sphere is used to express the DOA of the incident plane wave, and $\mathrm{o}$ is in the center of the array, which is the origin of the coordinate system. Consequently, it is taken as a reference point. In addition, when the incident signal direction is $(\alpha, \theta)$, azimuth $\alpha$ is expressed as the angle between the $x$-axis and a projection in the $x-y$ plane, and the projection is wired from the reference point to the source of the signal. The pitch angle $\theta$ is the angle between the $z$ axis and the wired one that is from the reference point to the source of signal. Then the delay time $\tau_{m}$ in which the signal arrives at the $m$ th element relative to the reference element is

$$
\tau_{m}=\frac{r}{c}\left(\cos \left(\frac{2 \pi(m-1)}{M}-\alpha\right) \sin \theta\right)
$$

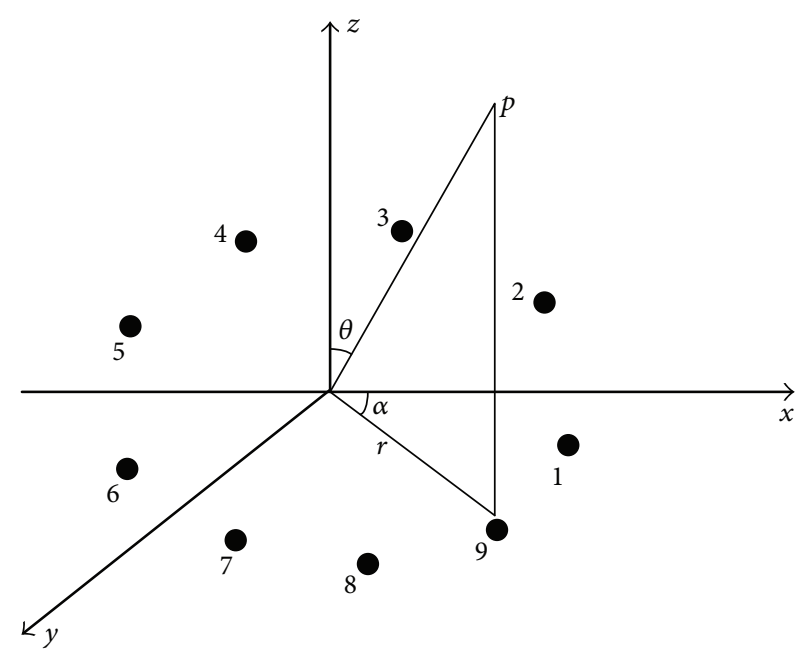

FIgURE 1: The structure of a nine-element circular ultrasonic array.

Then, according to (10) and (11), the steering vector of an $m$-element circular array can be expressed as

$$
\begin{aligned}
& \mathbf{a}(\alpha, \theta, f) \\
& =\left\{\begin{array}{c}
\exp \left[-j 2 \pi f \cos \alpha \frac{\sin \theta r}{c}\right] \\
\exp \left[-j 2 \pi f \cos \left(\frac{2 \pi}{M}-\alpha\right) \frac{\sin \theta r}{c}\right] \\
\vdots \\
\exp \left[-j 2 \pi f \cos \left(\frac{2 \pi(m-1)}{M}-\alpha\right) \frac{\sin \theta r}{c}\right]
\end{array}\right\},
\end{aligned}
$$

where the frequency $f$ is the whole frequency band of the signal.

\section{DOA Estimation Based on Sparse Decomposition}

3.1. The Mathematical Expression of Sparse Representation. Given an overcomplete dictionary $\mathbf{D}=\left\{\Phi_{i}, i=1,2, \ldots, I\right\}$, there are $I$ atoms, which is a whole Hilbert space $H=R^{d}$, and $I>d$. Therefore, for any signal expressed as $y, y \in H$, the $k$ atoms can be selected adaptively in $\mathbf{D}$, to make sparse approximation with the signal $y$; that is,

$$
y=\sum_{r \in I_{k}} c_{r} \Phi_{r}
$$

where $I_{k}$ is index set of $\Phi_{r}$ and the corresponding coefficients are expressed as $C=\left\{c_{r}\right\}_{r \in I_{k}}$. The atomic number $k$ selected is usually much smaller than the atomic number $I$ in the atom dictionary. A few atoms can express the signal, so-called sparse representation.

The matrix is used to express $y \in R^{d}$ and $\mathbf{D} \in R^{d \times I}$; the $i$ th column of $\mathbf{D}$ is $\Phi_{i}$, and then (13) can be written as

$$
\mathbf{Y}=\mathbf{D c} \text {, }
$$

where $\mathrm{c} \in R^{I}$ is a sparse vector. 
The ways in which the atoms in the overcomplete dictionary are used to express the signal have infinite variety of forms. Therefore, how to effectively solve the sparse coefficient vector $\mathbf{c}$ is an important problem, the sparse representation which is the basic problem of sparse representation, and the specific expression is as follows:

$$
\begin{aligned}
\operatorname{argmin} & \left\|\mathbf{c}_{0}\right\|, \\
\text { s.t. } & y=\mathbf{D c},
\end{aligned}
$$

where $\mathbf{c}_{0}$ is $l_{0}$-norm of $\mathbf{c}$, which is the number of the nonzero elements in the coefficient vector $\mathbf{c}$.

\subsection{Application of Matching Pursuit Algorithm in DOA Esti-} mation. In the application process of sparse representation method, different overcomplete dictionaries are constructed according to different research purposes. When an ultrasonic array is used to estimate the DOA of the broadband PD signal, the overcomplete dictionary can be structured according to a steering vector matrix form of the received signal. The steering vector matrix contains the wave direction of signals; therefore, constructing a group of atomic vectors covered space at any angle inevitably includes the DOA of signals. Based on sparse representation theory, these atoms that include the DOA of signals can be selected by using matching pursuit (MP) algorithm, and they can be used to realize the direction finding.

The principles of the MP algorithm are similar to the adaptive projection decomposition algorithm. Firstly, the atoms that match with the signal mostly are selected from the overcomplete dictionary, which is the idea that these atoms have the maximum inner product with signal. Here, the projection coefficient is that the signal on the atom is the largest, and the rest of energy on the atom after decomposition is minimum. Next, the same method is used to find out the best matching atoms with the remaining amount and then make decomposition. Repeat the above steps. When the remaining energy of decomposition is small enough or the best matching atom combinations can represent the original signal, stop the decomposition. The flow chart of the sparse representation by using MP algorithm is shown in Figure 2.

When estimating the DOAs of the ultrasonic signal generated by $p$ PD sources, the incidence angles of the signal can be searched on $N$ angle vectors that have been set. In general, the number of the PD sources is much smaller than the number of angles to be searched; that is, $P \ll N$. A search vector of the angle is constructed, and the vector is covering all space angle, which is $\beta=\left[\begin{array}{llll}\beta_{1} & \beta_{2} & \cdots & \beta_{N}\end{array}\right]$. There are $P$ components equal to $\varphi_{1}, \varphi_{2}, \ldots, \varphi_{P}$, respectively. According to (9), the search matrix of angle is constructed as

$$
A_{s}(\beta)=\left[a_{v}\left(\beta_{1}\right), a_{v}\left(\beta_{2}\right), \ldots, a_{v}\left(\beta_{N}\right)\right] .
$$

The direction finding by using the sparse representation theory is to decompose the received signals on the atoms with different directions. The projection value is maximum when the incident signal has the same direction with the atoms. According to the relevant knowledge of the vector projection theory in mathematics, the projection of an array

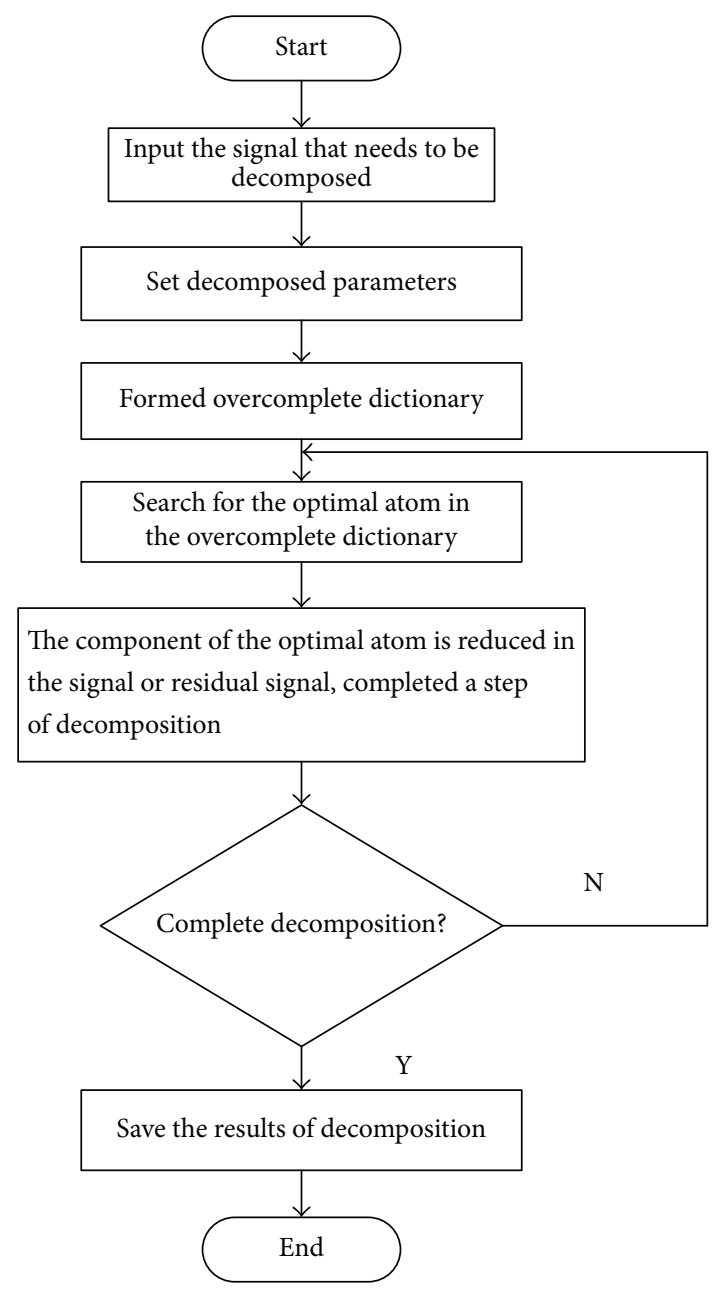

FIGURE 2: The flow chart of sparse representation based on MP algorithm.

signal on the atom is maximum, which means that the inner product module between the array signal and the corresponding atom is maximum. Firstly, the parameter to be decomposed is set to be $\mathbf{X}$, and $\mathbf{X}$ makes the inner product with each atom $\boldsymbol{\alpha}_{v}\left(\beta_{n}\right)(n=1,2, \ldots, N)$; then the optimal atom $a_{v}\left(\beta_{\gamma_{0}}\right) \gamma 0 \in\{1,2, \ldots, P\}$ is selected by the absolute value of the inner product, and the optimal atom meets the following conditions:

$$
\left|\left\langle\mathbf{X}, \mathbf{a}_{v}\left(\beta_{\gamma 0}\right)\right\rangle\right|=\sup \left|\left\langle\mathbf{X}, \mathbf{a}_{v}\left(\beta_{n}\right)\right\rangle\right|
$$

The received signal $\mathbf{X}$ is decomposed into the component of projection on $\mathbf{a}_{v}\left(\beta_{\gamma 0}\right)$ and the remains of the signal:

$$
X=P_{a_{v}\left(\beta_{\gamma 0}\right)} X+R X
$$

where $P_{a_{v}\left(\beta_{\gamma 0}\right)} X$ is the projection of signal on the optimal atom. And with the definition of the matrix projection, the 
part of projection can be obtained by using the following equation:

$$
\begin{aligned}
& \mathbf{P}_{a_{v}\left(\beta_{\gamma 0}\right)} \mathbf{X} \\
& \quad=\mathbf{a}_{v}\left(\beta_{\gamma 0}\right)\left\langle\mathbf{a}_{v}\left(\beta_{\gamma 0}\right), \mathbf{a}_{v}\left(\beta_{\gamma 0}\right)\right\rangle^{-1} \mathbf{a}_{v}{ }^{H}\left(\beta_{\gamma 0}\right) \mathbf{X} \\
& \quad=\left\langle\mathbf{X}, \mathbf{a}_{v}\left(\beta_{\gamma 0}\right)\right\rangle \mathbf{a}_{v}\left(\beta_{\gamma 0}\right) .
\end{aligned}
$$

Repeat the above steps with the residual signal and, after the decomposition for $\mathbf{P}$ times, the residual signal is small enough to meet the requirements of the allowable error, so the decomposition results of the array signal $\mathbf{X}$ can be obtained:

$$
\mathbf{X}=\sum_{n=1}^{P}\left\langle\mathbf{R}_{n} \mathbf{X}, \mathbf{a}_{v}\left(\beta_{n}\right)\right\rangle \mathbf{a}_{v}\left(\beta_{n}\right)+R_{k} \mathbf{X} .
$$

When the decomposition of the received signal has been finished, a group of orientation matrixes $\beta=\left[\begin{array}{llll}\beta_{1} & \beta_{2} & \cdots & \beta_{P}\end{array}\right]$ can be obtained. And $P$ elements are wave directions of $P$ signals, respectively.

The number of PD sources is previously unknown and, according to the signal sparse representation in the process of the change in energy, the iterative termination conditions for DOA estimation of ultrasonic array signals based on MP algorithm are obtained. However, if the difference of the energy variation for the adjacent decomposition is particularly large and the value of the energy variation is small in the process of subsequent classification, then the iteration can be terminated.

3.3. The Principle of Direction Finding Based on Sparse Representation of the Eigenvectors. According to the introduction of Section 2.1, assuming that the signal and noise are independent of each other, the center frequency is $f$, the array covariance matrix of received data is

$$
\begin{aligned}
\mathbf{R}(f) & =E\left\{\mathbf{X}(f) \mathbf{X}^{H}(f)\right\} \\
& =\mathbf{A}(f) \mathbf{R}_{S}(f) \mathbf{A}^{H}(f)+\delta^{2} \mathbf{I},
\end{aligned}
$$

where $\mathbf{I}$ is identity matrix, $\sigma^{2} \mathbf{I}=E\left[\mathbf{N}(f) \mathbf{N}(f)^{H}\right]$, and $\mathbf{R}_{S}(f)$ is the covariance matrix of the source signal. Moreover, the signal subspace composed by the signal eigenvector and the noise subspace composed by the noise eigenvector can be obtained, respectively, by the decomposition of the covariance of the ultrasonic array signal.

Theorem 1. Suppose that $N(N \leq M-1)$ narrowband farfield signal is incident on the array that consists of $M$ elements, the order of the array manifold matrix is $N$, and the order of the signal covariance matrix is $K(K \leq N)$. Assuming that the noise covariance matrix $\mathbf{R}_{N}$ is a matrix with full rank, so the following linear relationship meets

$$
\mathbf{R}_{N} \mathbf{e}_{k}=\sum_{n=1}^{N} \boldsymbol{\alpha}_{k}(n) \mathbf{a}\left(\theta_{n}\right),
$$

where $1 \leq k \leq K, \mathbf{e}_{k}$ is an eigenvector of covariance matrix that receives the data, $\boldsymbol{\alpha}_{k}(n)$ is a factor of linear combination, and $\mathbf{a}\left(\theta_{n}\right)$ is a steering vector. The proof process is in the literature [21].

Based on the theorem, when the noise covariance matrix is the ideal white noise, (22) can be simplified as

$$
\mathbf{e}_{k}=\sum_{n=1}^{N} \boldsymbol{\alpha}_{k}(n) \mathbf{a}\left(\theta_{n}\right) \quad 1 \leq k \leq K .
$$

Equation (23) shows that whether the source of signal is coherent, the eigenvectors corresponding to the maximum eigenvalue is a linear combination of the steering vectors for each signal source. And the biggest eigenvector of the data covariance matrix contains the information of all signals.

Consequently, the eigenvector corresponding to the maximum eigenvalue can be sparse representation, thereby the DOA estimation for the signal is obtained. Compared with the DOA estimation of the received data based on sparse representation, the eigenvalue decomposition can weaken the interference caused by noise, and the eigenvector corresponding to the maximum eigenvalue is selected to be as the amount to be decomposed, and the estimation results will be more accurate.

3.4. The Steps of Direction Finding Based on Sparse Representation of Eigenvectors. Firstly, in order to obtain the narrow covariance matrix of a single frequency, the received data need to be focused on, because the ultrasonic signal is a broadband signal. The rotate signal subspace (RSS) algorithm presented in literature [20] is used to focus on the received data of the array in this work.

Therefore, the detailed steps of the DOA estimation based on sparse representation of eigenvectors can be expressed as follows:

(1) The data $\mathbf{X}$ received by the ultrasonic array sensor is analyzed based on the DFT to obtain the $\mathbf{X}^{\prime}$, and this is the preparation for the subsequent focus.

(2) The reference frequency is selected as $f_{0}$, and $\mathbf{X}^{\prime}$ is focused by RSS, and the covariance matrix of a single frequency $\mathbf{P}$ is obtained by using the focus algorithm.

(3) The corresponding eigenvector $\mathbf{e}_{\max }$ of the maximum eigenvalue can be obtained through the eigenvalue decomposition of the covariance matrix $\mathbf{P}$.

(4) According to Section 2.2, the overcomplete dictionary is established in the form of the steering vector, and the frequency $f$ of the atom in the step and step overcomplete dictionary is replaced with the reference frequency $f_{0}$.

(5) By using the MP algorithm, the eigenvector $\mathbf{e}_{\max }$ is to make sparse representation, and the optimal atom is selected. Then the DOA estimation of the signal is obtained, which is contained in the angle $(\alpha, \theta)$ of the optimal atom.

3.5. Three-Array Cross Localization Method. After the DOA estimation of the signal, the position of the PD source cannot be sure, because the distance between the PD source and the 


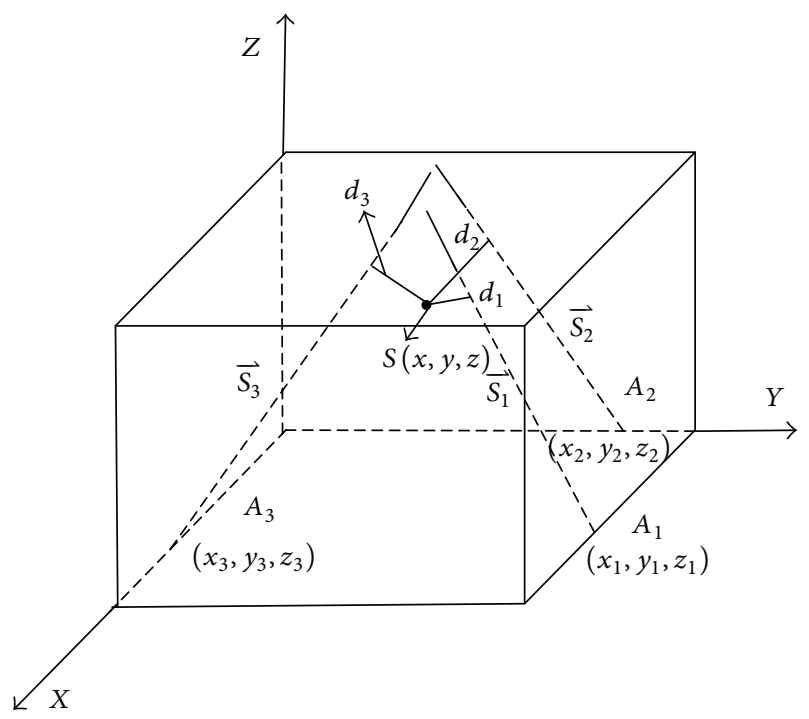

FIGURE 3: The map of three-array cross localization principle.

array sensor is unknown $[22,23]$. The space position of the PD source is obtained according to the method of three-array cross localization method and the results of the direction finding. The principle of three-array localization method is shown in Figure 3.

The space positions of the three sets of array sensors are, respectively, $A_{1}\left(x_{1}, y_{1}, z_{1}\right), A_{2}\left(x_{2}, y_{2}, z_{2}\right)$, and $A_{3}\left(x_{3}, y_{3}, z_{3}\right)$ and, using the direction angle and the positions of the ultrasonic array sensor, the equation of the direction line can be obtained. Suppose that spatial coordinates of the signal source are $S(x, y, z)$. In the ideal situation, the three different direction lines should intersect in the $S(x, y, z)$. But these lines are on different surfaces, because there are many actual measurement errors. Therefore, the sum function that is a sum of the vertical distance from a point in the space to the three lines is

$$
d=\sum_{t=1}^{3} d_{t}=d_{1}+d_{2}+d_{3}
$$

Through searching in the space by using Chaotic Monkey algorithm [24], when the sum of distance is minimum, the point with the minimum value $d$ can be regarded as the space position of the PD source.

\section{The Simulation Study}

4.1. The Simulation of the PD Signal. The length $(x)$, width $(y)$, and height $(z)$ of the electrical equipment model are, respectively, $150 \mathrm{~cm}, 100 \mathrm{~cm}$, and $120 \mathrm{~cm}$ (they are matched with the size of the experimental equipment). The simulation parameters of signal are set as the wavelength $\lambda=10 \mathrm{~mm}$; the amplitude is $5 \mathrm{~mm}$; the center frequency is $150 \mathrm{kHz}$; the equivalent velocity is $1500 \mathrm{~m} / \mathrm{s}$; the acoustic attenuation coefficient of the signal is $\alpha=50 \times 10^{-7} \mathrm{~cm}^{-1}$; the number of sampling snapshots is 1024; the sampling frequency is $2 \mathrm{MHz}$; the noise-signal ratio is $10 \mathrm{~dB}$. Moreover, in order to verify the validity of the array signal direction finding based on sparse representation, the simulation research is carried out on a nine-element circular ultrasonic array sensor, and the interval between array elements is $d=\lambda / 2=5 \mathrm{~mm}$.

The form of simulated signal $[25,26]$ is

$$
f(t)= \begin{cases}A e\left(k_{1}\left(t_{0}-t\right)\right) \cos (2 \pi f t), & 0 \leq t \leq t_{0} \\ A e\left(k_{2}\left(t-t_{1}\right)\right) \cos (2 \pi f t), & t_{0} \leq t \leq t_{1}\end{cases}
$$

where $f$ is the central frequency of the signal, $A$ is the amplitude of the signal, and $t_{0}$ is the time division point. Firstly, because of the randomness, the PD ultrasonic signal is in electrical equipment, so 100 frequency points of the signal are generated according to the average probability in the bandwidth, and they formed frequency distribution. Then, on the basis of center frequency of ultrasonic signal, the amplitude of the signal corresponding to each frequency point is formed by the normal distribution method. Finally, the initial phase of each frequency point is randomly generated, and the white Gaussian noise is added in the signal, and the PD ultrasonic signal in the oil can be simulated.

The map of the time waveform of the simulated signal is shown in Figure 4(a); by using the Fourier transform, the map of the frequency domain is shown in Figure 4(b); when the noise is large, the PD signal is submerged in the waveform of the time domain, and it is shown in Figure 4(c).

The map of the frequency domain shows that the simulated PD signal is a broadband signal, and the center frequency is $150 \mathrm{kHz}$.

The oscillogram of the simulated signal received by a nine-element circular ultrasonic array sensor is shown in Figure 5.

4.2. The Simulation of Location. For the broadband signal simulated, the received data by ultrasonic array sensor is segmented according to the observation time, and the array covariance matrix of each frequency point can be obtained by the DFT in every period. $f_{0}$ is selected as focusing frequency, and the covariance matrix of a single frequency can be acquired. The step and step overcomplete dictionary is established according to the focusing frequency and the steering vector form of Section 2.2. After the focusing and the eigendecomposition of the covariance matrix, the eigenvector corresponding to the maximum eigenvalue can be acquired, and the eigenvector is the parameter to be decomposed.

Then taking a circular ultrasonic array sensor, for example, the position of the source is set at $(35,50,60) \mathrm{cm}$, and the positions of the three-array sensors are set at position $\# 1$ : $(40,0,10) \mathrm{cm}$, position \#2: $(80,0,0) \mathrm{cm}$, position \#3: $(0$, $30,50) \mathrm{cm}$. Therefore, the theoretical values of the DOAs are, respectively, $\left(5.7^{\circ}, 45.1^{\circ}\right),\left(132.0^{\circ}, 48.3^{\circ}\right)$, and $\left(29.7^{\circ}, 76.1^{\circ}\right)$.

In accordance with the steps of the Section 3.4, the process of searching for the optimal atom is that the array signal makes inner products with each atom, respectively, and the value of the inner product is maximum with the optimal atom. In order to figuratively present this process, the scatter gram of absolute value of the inner product in the angle space can be made. 


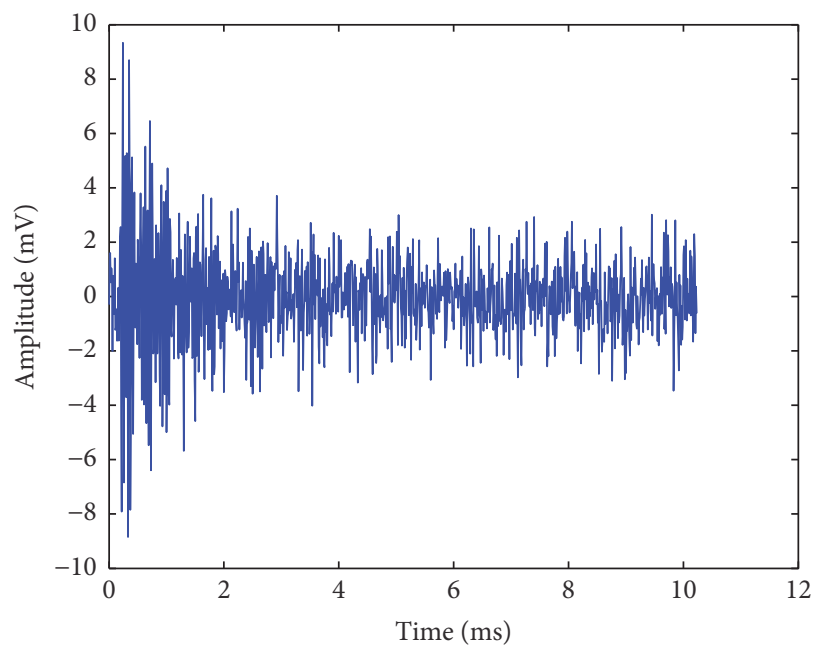

(a) The waveform of the time domain

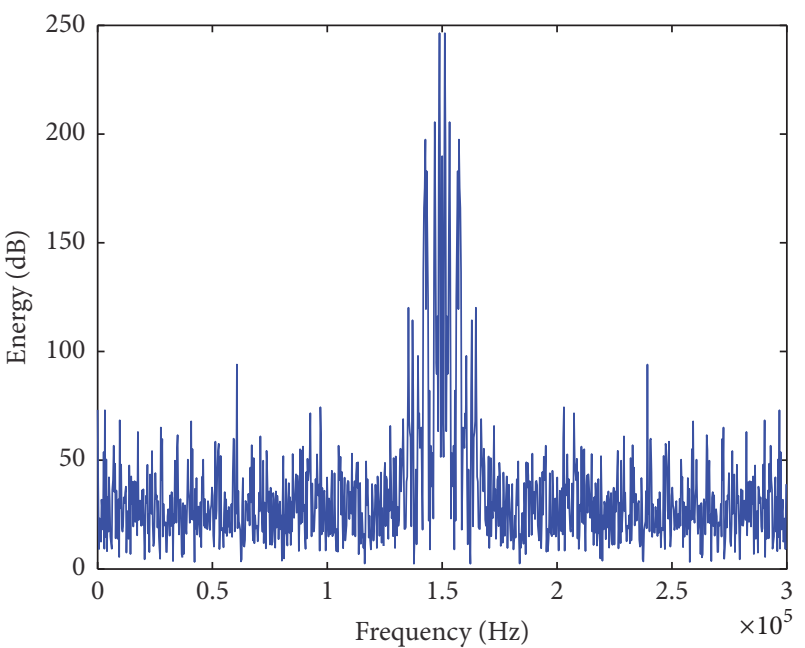

(b) The waveform of the frequency domain

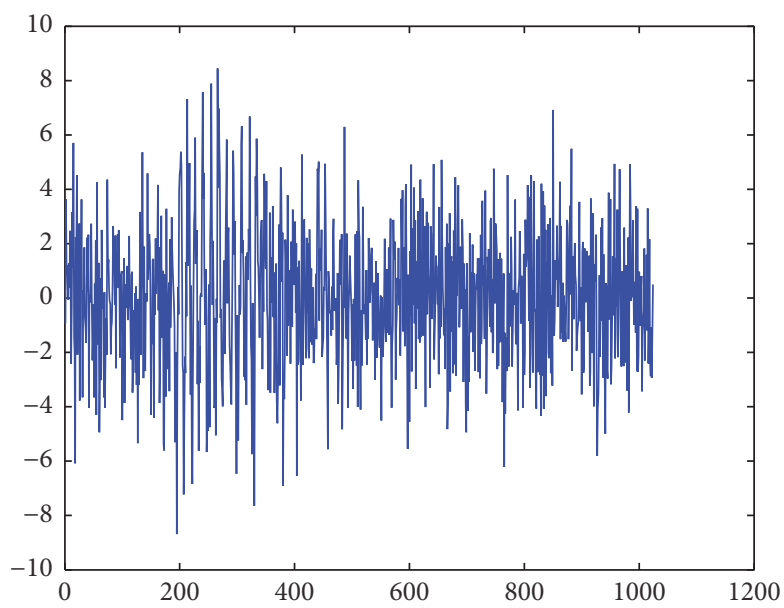

(c) The PD signal with the noise

FIgURE 4: The simulated PD signal.

The DOA estimation result of the PD ultrasonic signal received by the array sensor at the position $\# 1$ is $\left(95.9^{\circ}, 4.8^{\circ}\right)$, and the scatter gram of the absolute value of the inner product in the space is shown in Figure 6.

The DOA estimation result of the PD ultrasonic signal received by the array sensor at position $\# 2$ is $\left(131.6^{\circ}, 8.5^{\circ}\right)$, and the scatter gram of the absolute value of the inner product in the space is shown in Figure 7.

The DOA estimation result of the PD ultrasonic signal received by the array sensor at position $\# 3$ is $\left(30.1^{\circ}, 6.4^{\circ}\right)$, and the scatter gram of the absolute value of the inner product in the space is shown in Figure 8.

Then using the three DOA estimation results above, the objective function equation (24) is calculated by the threearray cross positioning principle. And when the objective function is minimum by using the search of the optimization algorithm, the position of the PD source in the space can be acquired. Consequently, the result is $(33.1,51.8,58.7) \mathrm{cm}$, the error is $2.9 \mathrm{~cm}$, and the location diagram is shown in Figure 9.
Changing the positions of the PD source and the ultrasonic array sensors, the five groups of the PD source positioning simulation are conducted. The positioning results of the circular ultrasonic array are shown in Table 1.

The table shows that, after direction of the eigenvector with the sparse representation, the average error for positioning is $3.08 \mathrm{~cm}$. And it illustrates that the eigenvector with the sparse representation can obtain the better direction finding results and reduce the errors in the positioning.

\section{The Experimental Study}

5.1. The Experimental System. The experimental system for research includes discharge device, the array sensor, the data acquisition system, and the data processing system.

The simulated electrical equipment is a tank welded by steel plates, the body length is $150 \mathrm{~cm}$, the width is $100 \mathrm{~cm}$, the height is $120 \mathrm{~cm}$, and the thickness of the steel plate is $5 \mathrm{~mm}$. 

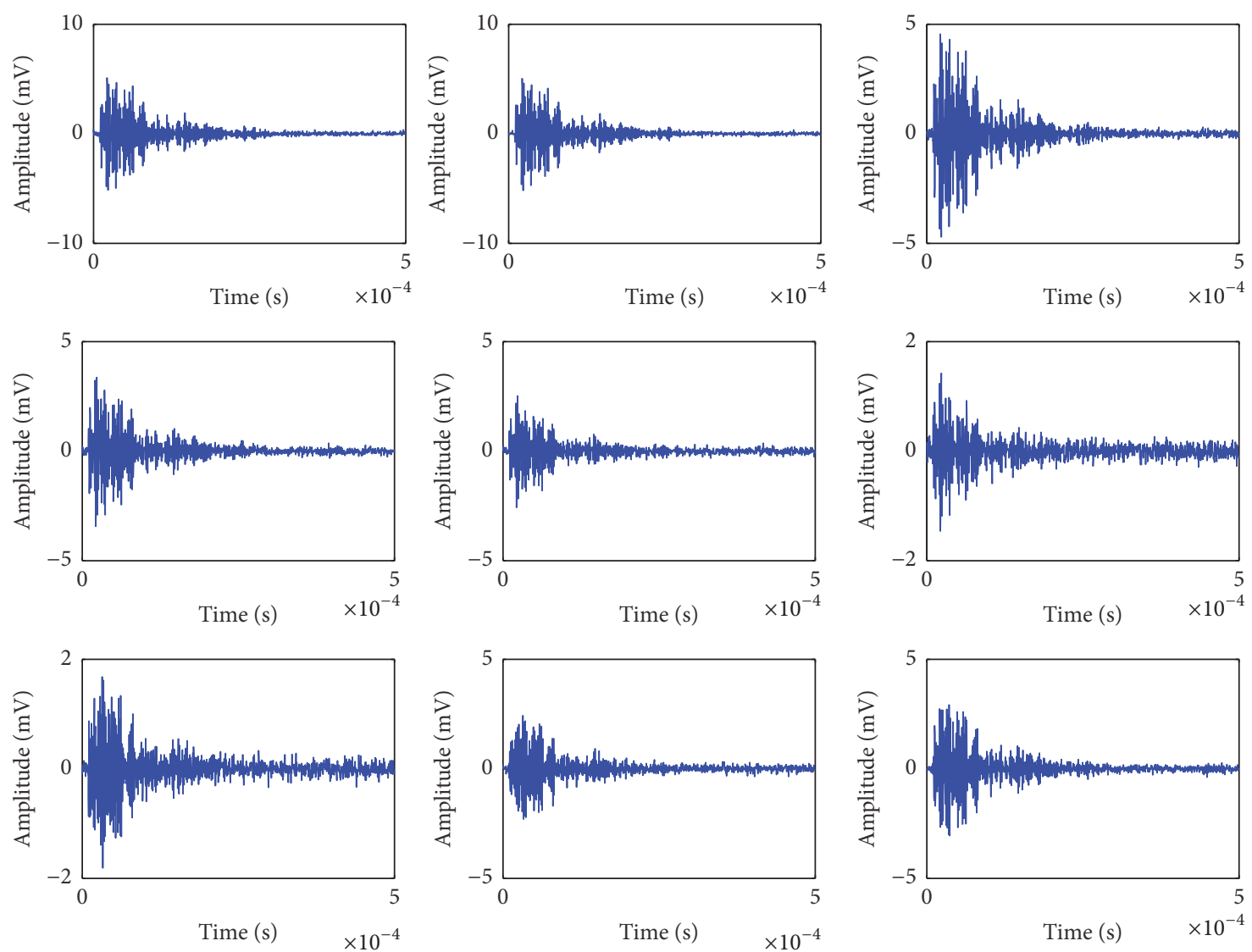

FIGURE 5: The oscillogram of the signal received by a full ultrasonic array.

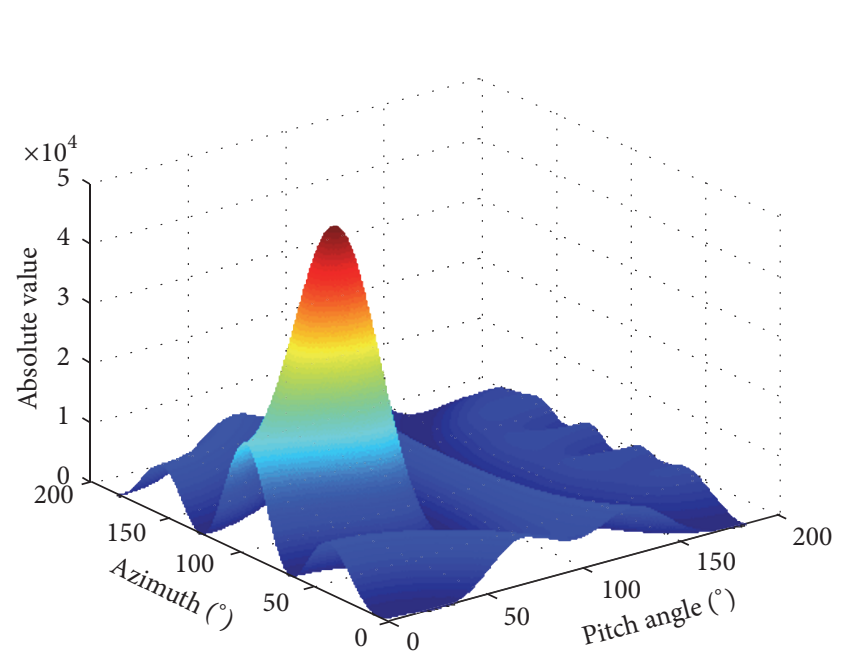

(a) The stereogram

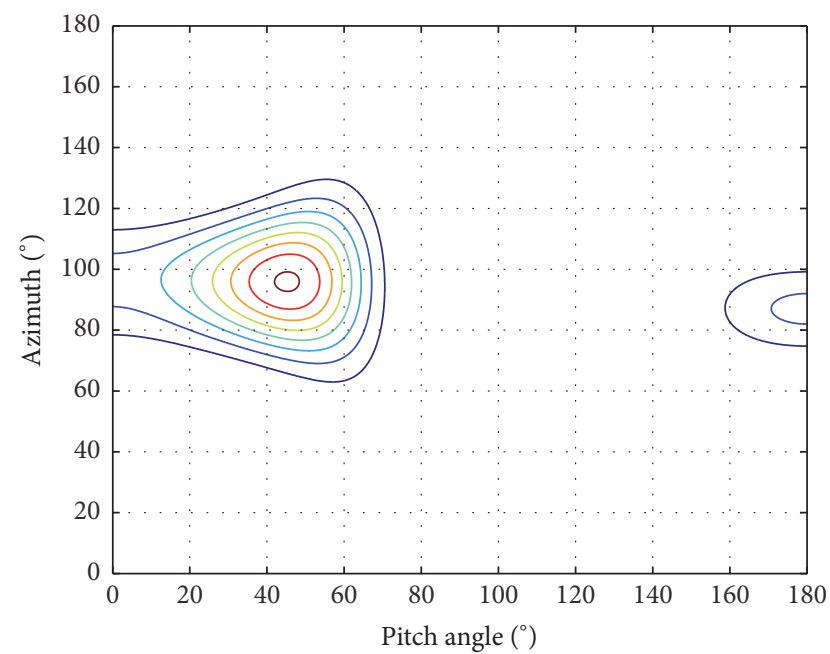

(b) The contour map

FIGURE 6: The scatter gram of the absolute value of the inner product in the space.

Moreover, a three-capacitor discharge tube is used to simulate the PD source of the internal electrical equipment. And the array sensor is put in the preset position.

A nine-element circular ultrasonic array sensor is used to receive signal, it is fixed on the outer wall of the tank, and the shielding lines are used to transfer the data; then the data are processed by the computer combined with ultrasonic detection software. The principle diagram of the whole experiment system is shown in Figure 10.

The scale model and the physical map of the nine-element circular ultrasonic array sensor are, respectively, shown in Figures 11(a) and 11(b). 


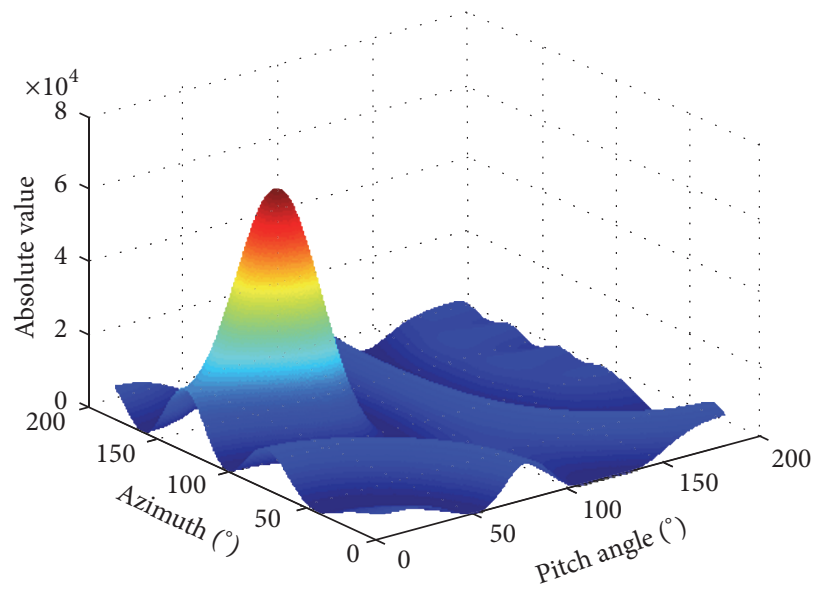

(a) The stereogram

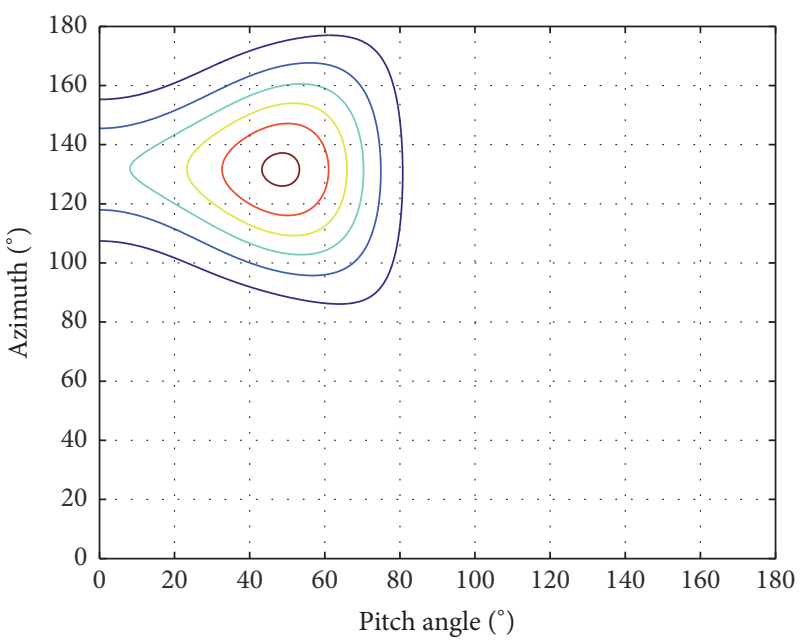

(b) The contour map

FIGURE 7: The scatter gram of the absolute value of the inner product in the space at position 2.

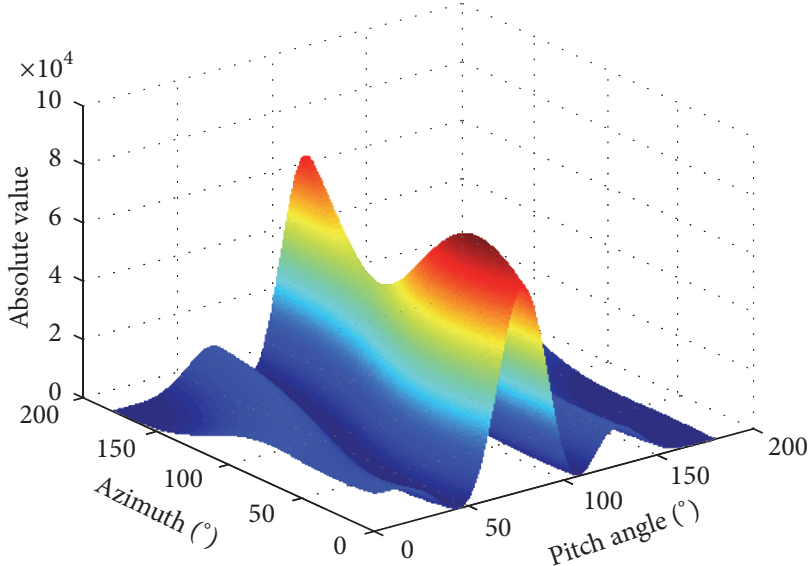

(a) The stereogram

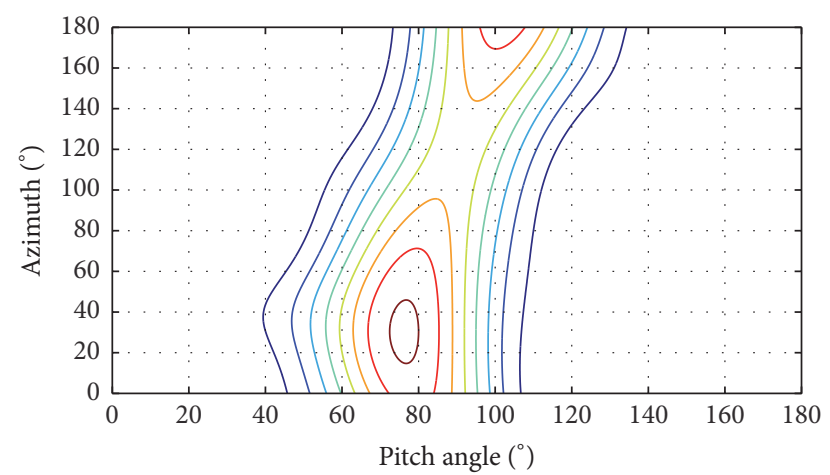

(b) The contour map

FIGURE 8: The scatter gram of the absolute value of the inner product in the space at position 3.

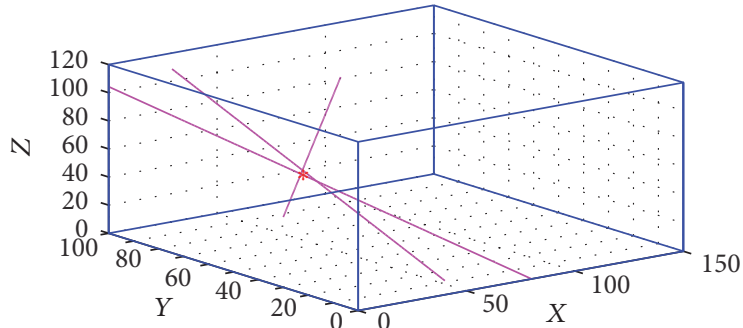

FIGURE 9: The map of simulated location of the circular array sensor. “*” refers to the position of the PD source.

In order to avoid the influences of discharge instability and other factors on the experimental results, a threecapacitor discharge tube is used to simulate the PD source, it can generate the ultrasonic that is similar to the ultrasonic signal of the real partial discharge, and it has good stability and repeatability, and the discharge voltage is low and easy to meet the insulation. The EPSON discharge tube is used to be a discharge device, and the critical discharge voltage is $230 \mathrm{~V}$. Generally, the discharge frequency of ultrasonic signals emitted by the discharge tube is in the range of $50 \mathrm{kHz}$ to $280 \mathrm{kHz}$, the center frequency is $150 \mathrm{kHz}$, and the equivalent velocity is $1500 \mathrm{~m} / \mathrm{s}$, while the wavelength is about $10 \mathrm{~mm}$. The diagram of the three-capacitor discharge principle is shown in Figure 12.

In Figure 12, $C_{0}$ is a coupling capacitor, and $C_{1}$ is equivalent capacitance of the other parts of the insulating medium. $\mathrm{C}_{2}$ is equivalent capacitance of the insulating medium that is in series with the PD source, and the gas-discharge tube is used to be a discharge device, when the voltage of the tube 
TABLE 1: The location results of the circular ultrasonic array.

\begin{tabular}{|c|c|c|c|c|c|c|}
\hline Group & $\begin{array}{c}\text { The position } \\
\text { of the PD } \\
\text { source/cm }\end{array}$ & $\begin{array}{l}\text { The position } \\
\text { of the array } \\
\text { sensor } / \mathrm{cm}\end{array}$ & $\begin{array}{c}\text { Theoretical } \\
\text { angle } /\left(^{\circ}\right)\end{array}$ & $\begin{array}{l}\text { Direction } \\
\text { angle } /\left(^{\circ}\right)\end{array}$ & $\begin{array}{l}\text { The result of the } \\
\text { three-array cross } \\
\text { positioning } \\
/ \mathrm{cm}\end{array}$ & The error/cm \\
\hline \multirow{3}{*}{1} & \multirow{3}{*}{$(35,50,60)$} & $(40,0,10)$ & $(95.7,45.1)$ & $(95.9,44.8)$ & \multirow{3}{*}{$(33.1,51.8,58.7)$} & \multirow{3}{*}{2.9} \\
\hline & & $(80,0,0)$ & $(132.0,48.3)$ & $(131.6,48.5)$ & & \\
\hline & & $(0,30,50)$ & $(29.7,76.1)$ & $(30.1,76.4)$ & & \\
\hline \multirow{3}{*}{2} & \multirow{3}{*}{$(30,80,95)$} & $(35,0,50)$ & $(93.6,60.7)$ & $(94.1,60.4)$ & \multirow{3}{*}{$(32.0,78.5,93.2)$} & \multirow{3}{*}{3.1} \\
\hline & & $(40,0,0)$ & $(97.1,40.3)$ & $(97.5,39.5)$ & & \\
\hline & & $(0,20,30)$ & $(63.4,45.9)$ & $(64.3,46.2)$ & & \\
\hline \multirow{3}{*}{3} & \multirow{3}{*}{$(25,70,20)$} & $(50,0,10)$ & $(109.7,82.3)$ & $(110.3,81.6)$ & \multirow{3}{*}{$(23.2,68.4,22.3)$} & \multirow{3}{*}{3.3} \\
\hline & & $(0,50,0)$ & $(38.7,58.0)$ & $(38.3,57.4)$ & & \\
\hline & & $(0,50,60)$ & $(38.7,141.3)$ & $(38.3,139.8)$ & & \\
\hline \multirow{3}{*}{4} & \multirow{3}{*}{$(60,30,50)$} & $(30,0,20)$ & $(45.0,54.7)$ & $(44.3,54.1)$ & \multirow{3}{*}{$(62.3,31.7,49.0)$} & \multirow{3}{*}{3.0} \\
\hline & & $(65,0,35)$ & $(99.5,63.7)$ & $(98.7,64.0)$ & & \\
\hline & & $(0,45,0)$ & $(166.0,51.0)$ & $(165.2,50.5)$ & & \\
\hline \multirow{3}{*}{5} & \multirow{3}{*}{$(75,20,45)$} & $(0,0,80)$ & $(14.9,114.3)$ & $(14.4,114.8)$ & \multirow{3}{*}{$(73.2,21.7,43.1)$} & \multirow{3}{*}{3.1} \\
\hline & & $(40,0,0)$ & $(29.7,41.9)$ & $(30.3,40.8)$ & & \\
\hline & & $(55,0,0)$ & $(45.0,32.2)$ & $(44.1,32.7)$ & & \\
\hline
\end{tabular}

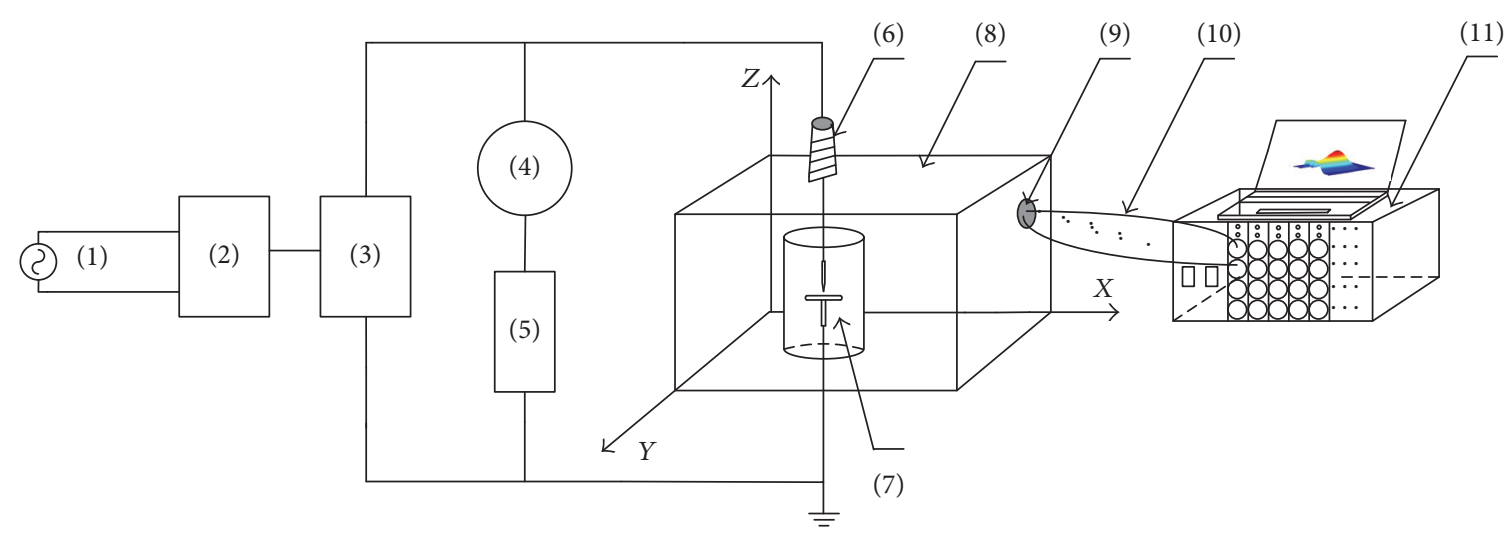

FIGURE 10: The structure of the PD positioning experimental system. (1) AC power regulator; (2) voltage regulator; (3) test transformer; (4) coupling capacitor; (5) impedance measurement; (6) inlet bushing; (7) PD model; (8) oil tank; (9) Partial Discharge Ultrasonic array sensor; (10) transmission lines; (11) data acquisition unit.

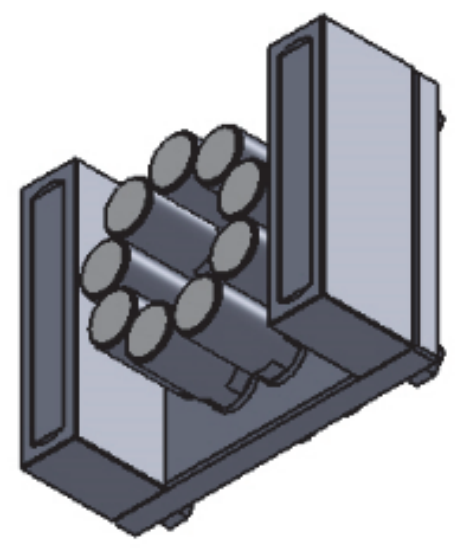

(a) The scale model

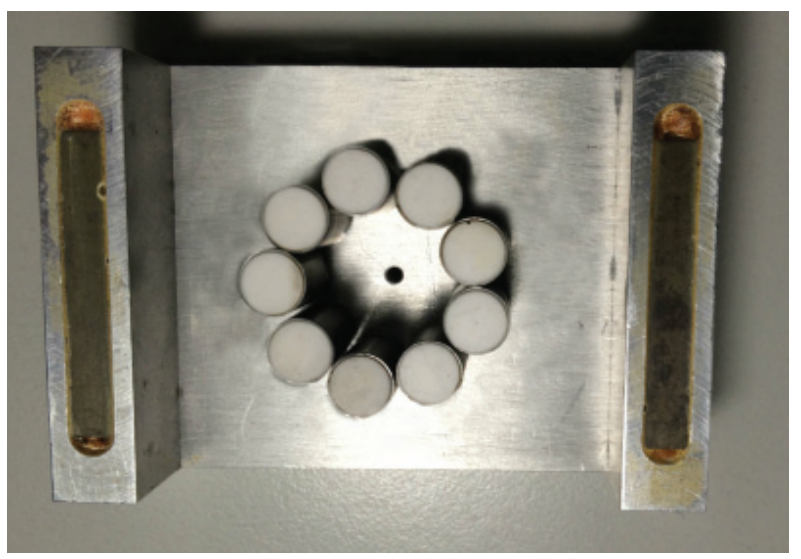

(b) The physical map

FIGURE 11: A nine-element circular ultrasonic array sensor. 
TABLE 2: The positioning results based on sparse representation of the eigenvector.

\begin{tabular}{|c|c|c|c|c|c|}
\hline Group & $\begin{array}{l}\text { The position } \\
\text { of the PD } \\
\text { source/cm }\end{array}$ & $\begin{array}{l}\text { Theoretical } \\
\text { angle } /\left(^{\circ}\right)\end{array}$ & $\begin{array}{l}\text { Direction } \\
\text { angle } /\left(^{\circ}\right)\end{array}$ & $\begin{array}{l}\text { The result of the } \\
\text { three-array cross } \\
\text { positioning/cm }\end{array}$ & The error $/ \mathrm{cm}$ \\
\hline \multirow{3}{*}{1} & $(45,0,20)$ & $(36.9,32.0)$ & $(36.0,31.2)$ & \multirow{3}{*}{$(67.7,17.4,62.6)$} & \multirow{3}{*}{4.5} \\
\hline & $(80,0,20)$ & $(135.0,27.9)$ & $(134.2,28.9)$ & & \\
\hline & $(0,30,40)$ & $(167.0,73.3)$ & $(168.1,73.5)$ & & \\
\hline \multirow{3}{*}{2} & $(55,0,35)$ & $(56.3,35.8)$ & $(55.2,36.6)$ & \multirow{3}{*}{$(62.5,12.2,57.1)$} & \multirow{3}{*}{4.7} \\
\hline & $(0,25,35)$ & $(163.0,69.8)$ & $(162.1,69.2)$ & & \\
\hline & $(45,0,20)$ & $(36.9,32.0)$ & $(37.5,32.9)$ & & \\
\hline \multirow{3}{*}{3} & $(0,45,45)$ & $(155.2,78.2)$ & $(154.0,79.4)$ & \multirow{3}{*}{$(62.0,17.6,62.2)$} & \multirow{3}{*}{4.5} \\
\hline & $(70,0,45)$ & $(108.3,46.5)$ & $(107.6,45.4)$ & & \\
\hline & $(45,0,40)$ & $(56.3,51.4)$ & $(55.6,52.0)$ & & \\
\hline \multirow{3}{*}{4} & $(55,0,45)$ & $(56.3,50.3)$ & $(55.5,51.2)$ & \multirow{3}{*}{$(67.4,12.5,62.7)$} & \multirow{3}{*}{4.4} \\
\hline & $(0,40,25)$ & $(158.9,63.3)$ & $(157.8,64.0)$ & & \\
\hline & $(45,0,20)$ & $(36.9,32.0)$ & $(37.5,31.1)$ & & \\
\hline \multirow{3}{*}{5} & $(55,0,35)$ & $(56.3,35.8)$ & $(57.2,35.0)$ & \multirow{3}{*}{$(67.8,17.6,62.4)$} & \multirow{3}{*}{4.5} \\
\hline & $(70,0,35)$ & $(108.3,32.3)$ & $(107.4,31.2)$ & & \\
\hline & $(0,40,20)$ & $(158.9,60.1)$ & $(159.7,61.2)$ & & \\
\hline
\end{tabular}

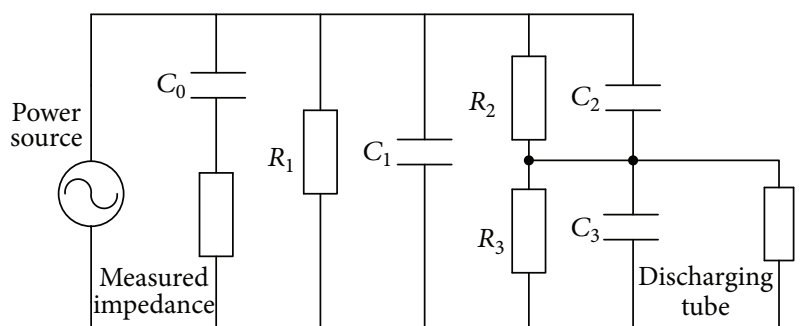

FIGURE 12: The diagram of the three-capacitor discharge principle.

reaches a certain value, the gas is breakdown conduction, and then the discharge effect is produced.

5.2. The Signal Processing. In the process of the experiment, the position of the PD source was preset. And a plurality of preset fixed locations were for the array sensors on the outer wall of the electrical equipment. Then the ultrasonic array sensor was used to collect the PD signal. In order to ensure that the experiment was performed under the same conditions and to facilitate subsequent data processing, the PD source location was fixed during the experiment, only changing the position of ultrasonic array sensor so that the relative space position was changed. The location of the PD source in experiment was $(65,15,60) \mathrm{cm}$.

The oscillogram of the $\mathrm{PD}$ signal received by the nineelement ultrasonic array sensor is shown in Figure 13.

Three different locations were selected to place ultrasonic array sensors that were used to receive the PD signal, and the ultrasonic signal was used to be focusing and the eigenvector corresponding to the maximum eigenvalue was acquired by using the eigendecomposition of the covariance matrix with a single frequency. This eigenvector was the amount to be decomposed, and the optimal atom was selected by using MP algorithm; then the DOA estimation can be realized. Besides, the noise interference can be reduced based on sparse representation of the eigenvectors and improve the accuracy of positioning.

Using the methods of direction finding and positioning in the work, the results are shown in Table 2.

The table shows that, in the experimental process, the average error of positioning for the circular ultrasonic array sensor based on sparse representation of the eigenvectors is $4.52 \mathrm{~cm}$, which meets the requirement of practical engineering.

\section{Conclusion}

The accurate detection of the PD in oil of electrical equipment is the key to the maintenance and repairs of equipment. The positioning method for the PD based on the sparse representation of the eigenvectors is studied in this work. First of all, the mathematical model of a wideband PD signal and the steering vector of a circular ultrasonic array sensor is given. Then the sparse representation theory is applied to the DOA estimation. The principle and process of the sparse presentation of the eigenvectors is introduced in detail, and the three-array cross positioning method is also introduced. Lastly, the simulation study and experimental research are conducted on this method, and the results show that the positioning method in the work can achieve the accurate positioning of the PD source. 

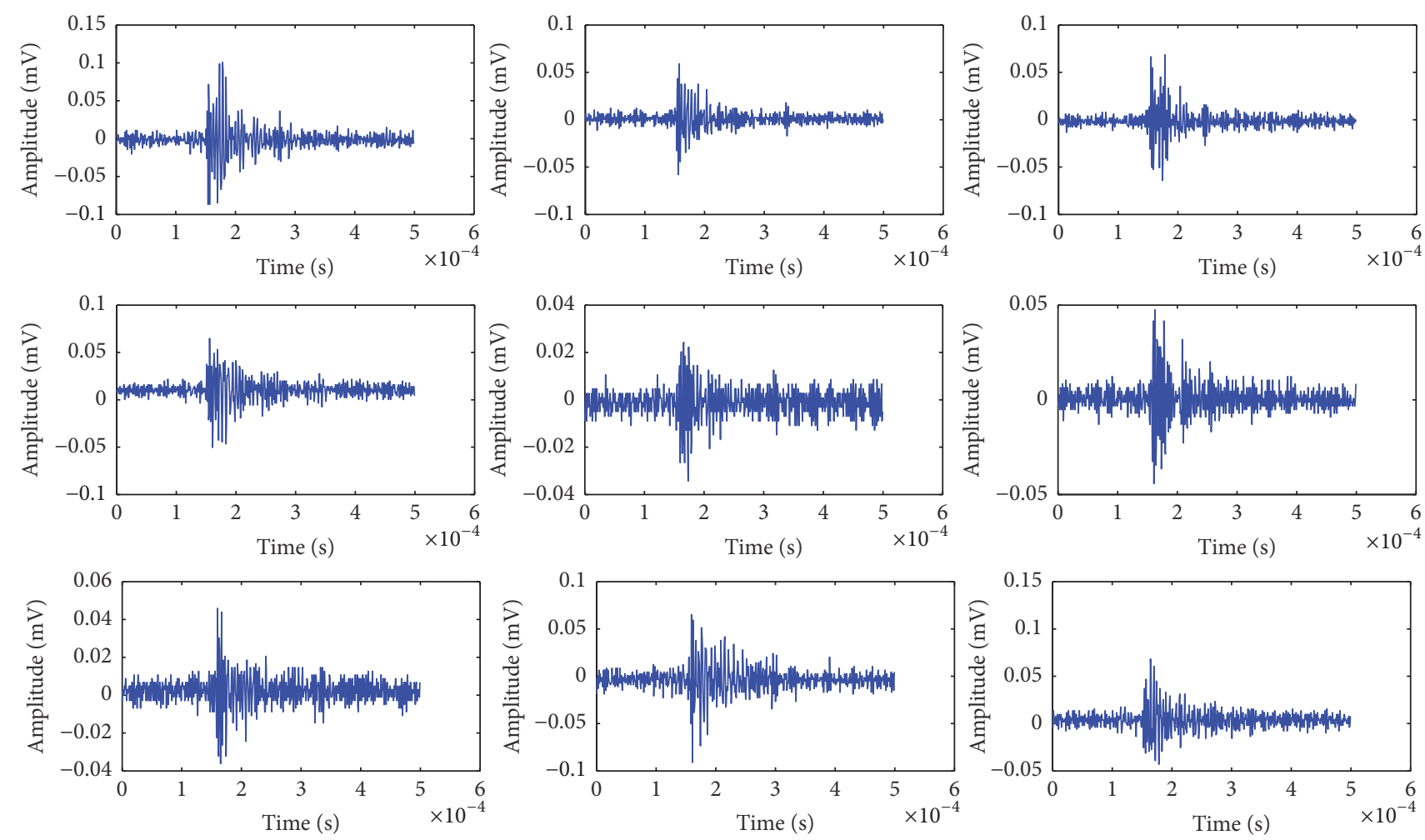

FIGURE 13: Nine channel ultrasonic signal waveforms.

\section{Competing Interests}

The authors declare that they have no competing interests.

\section{Acknowledgments}

This work was supported by Project Supported by National Natural Science Foundation of China (51307060); Project Supported by Natural Science Foundation of Hebei Province (E2015502081); State Key Laboratory of Alternate Electrical Power System with Renewable Energy Source (LAPS16009).

\section{References}

[1] C. R. Qiu and N. Q. Wang, Electrician Equipment Local Discharge and Its Test Technology, Mechanical Industry Publishing House, Beijing, China, 1994.

[2] Z. Liu, The Ultra-High Voltage Grid, China Electric Power Press, Beijing, China, 2005.

[3] J. Y. Wang, R. J. Liao, Y. Y. Zhang, and F. J. Meng, "Economic life assessment of power transformers using an improved model," CSEE Journal of Power and Energy Systems, vol. 1, no. 3, pp. 6875, 2015.

[4] W. J. Chen and X. Cui, "Foreword for the special section on AC and DC ultrahigh voltage technologies," CSEE Journal of Power and Energy Systems, vol. 1, no. 3, pp. 1-2, 2015.

[5] Q. Xie, S. Cheng, F. Lü, and Y. Li, "A new sparse design method on phased array-based acoustic emission sensor for partial discharge detection," Measurement Science \& Technology, vol. 25, no. 3, Article ID 035102, 11 pages, 2014.
[6] S. Chen, F. Lv, Q. Xie et al., "The transformer partial discharge positioning method based on transient voltage to earth and ultrasonic array signals," Transactions of China Electrotechnical Society, vol. 27, no. 4, pp. 255-262, 2012.

[7] Y. L. Wang, H. Chen, Y. N. Peng et al., The Spatial Spectrum Estimation Theory and Algorithms, Tsinghua University Press, 2004.

[8] E. Skudrzyk, The Foundations of Acoustics, Springer, New York, NY, USA, 1971.

[9] Q. Xie, S. Cheng, F. Lü, and Y. Li, "Location of partial discharge in transformer oil using circular array of ultrasonic sensors," IEEE Transactions on Dielectrics and Electrical Insulation, vol. 20, no. 5, pp. 1683-1690, 2013.

[10] Q. Xie, X. Liu, J. Tao, T. Li, S. Cheng, and F. Lu, "Experimental verification of the sparse design of a square partial discharge acoustic emission array sensor," Measurement Science \& Technology, vol. 26, no. 4, Article ID 045101, 2015.

[11] M. R. Rao and B. P. Singh, "Detection and localization of interturn fault in the $\mathrm{HV}$ winding of a power transformer using wavelets," IEEE Transactions on Dielectrics and Electrical Insulation, vol. 8, no. 4, pp. 652-657, 2001.

[12] M. Wax, T. J. Shan, and T. Kailath, Spatio-Temporal Spectral Analysis by Eigenstructure Methods, Stanford Univ Ca Information Systems Lab, 1984.

[13] S. Valaee, B. Champagne, and P. Kabal, "Localization of wideband signals using least-squares and total least-squares approaches," IEEE Transactions on Signal Processing, vol. 47, no. 5, pp. 1213-1222, 1999.

[14] S. Valaee and P. Kabal, "Wideband array processing using a twosided correlation transformation," IEEE Transactions on Signal Processing, vol. 43, no. 1, pp. 160-172, 1995. 
[15] S. Valaee and P. Kabal, "The optimal focusing subspace for coherent signal subspace processing," IEEE Transactions on Signal Processing, vol. 44, no. 3, pp. 752-756, 1996.

[16] M. Allam and A. Moghaddamjoo, "Two-dimensional DFT projection for wideband direction-of-arrival estimation," IEEE Transactions on Signal Processing, vol. 43, no. 7, pp. 1728-1732, 1995.

[17] M. A. Doron and A. J. Weiss, "On focusing matrices for wideband array processing," IEEE Transactions on Signal Processing, vol. 40, no. 6, pp. 1295-1302, 1992.

[18] S. G. Mallat and Z. Zhang, "Matching pursuits with timefrequency dictionaries," IEEE Transactions on Signal Processing, vol. 41, no. 12, pp. 3397-3415, 1993.

[19] S. Mallat and Z. Zhang, "Adaptive time-frequency decomposition with matching pursuits," in Proceedings of the IEEESP International Symposium Time-Frequency and Time-Scale Analysis, pp. 7-10, Victoria, Canada, October 1992.

[20] H. Hung and M. Kaveh, "Focussing matrices for coherent signal-subspace processing," IEEE Transactions on Acoustics, Speech, and Signal Processing, vol. 36, no. 8, pp. 1272-1281, 1988.

[21] J. A. Cadzow, Y.-S. Kim, and D.-C. Shiue, "General direction-ofarrival estimation: a signal subspace approach," IEEE Transactions on Aerospace \& Electronic Systems, vol. 25, no. 1, pp. 31-47, 1989.

[22] Q. Xie, J. Tao, Y. Wang, J. Geng, S. Cheng, and F. Lü, "Use of ultrasonic array method for positioning multiple partial discharge sources in transformer oil," Review of Scientific Instruments, vol. 85, no. 8, Article ID 084705, 2014.

[23] Q. Xie, Y.-Q. Li, F.-C. Lü, C.-R. Li, N. Wang, and Y.-J. Ding, "Method for PD location in oil combining ultrasonic phased array with wideband array signal processing," Proceedings of the Chinese Society of Electrical Engineering, vol. 29, no. 28, pp. 1317, 2009.

[24] Y. Q. Xu, H. W. Zhang, T. Li, Y. Yang, J. Tao, and Q. Xie, "Application of chaotic monkey algorithm in partial discharge location with ultrasonic array signals," Insulating Materials, vol. 47, no. 5, pp. 92-95, 2014.

[25] T. Wang, G. Cheng, and S. Wan, "Fourier-wavelet regularized deconvolution in medical ultrasound imaging," Technical Acoustics, vol. 30, no. 6, pp. 501-504, 2011.

[26] Z. Tan, D. Feng, G. Chen et al., "Pre-processing in medical ultrasonic lesions images," Foreign Electronic Measurement Technology, vol. 33, no. 3, pp. 89-91, 2014. 


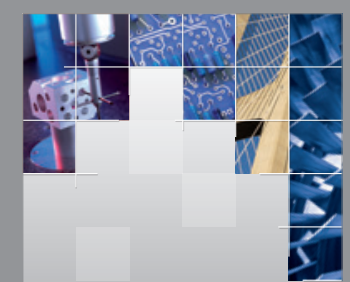

\section{Enfincering}
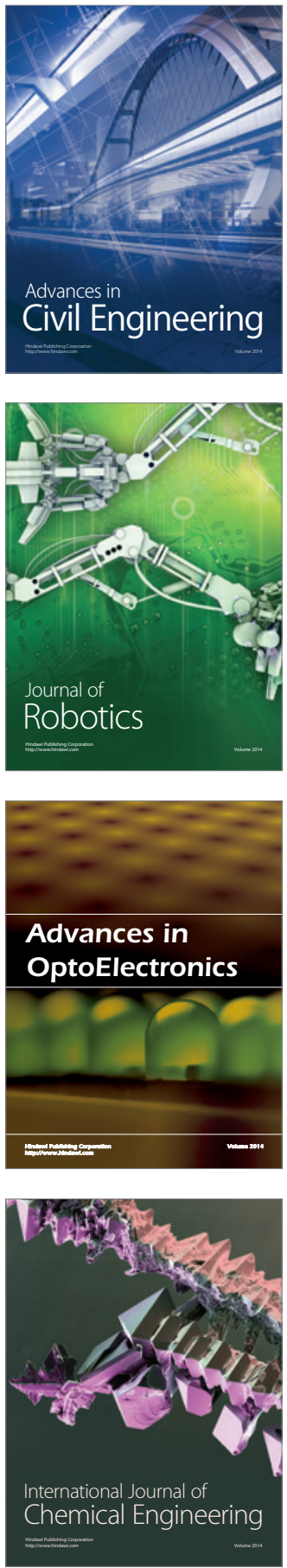

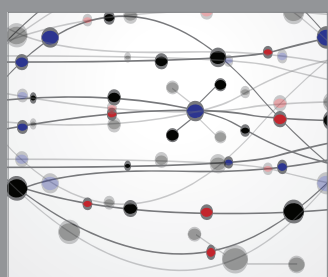

The Scientific World Journal

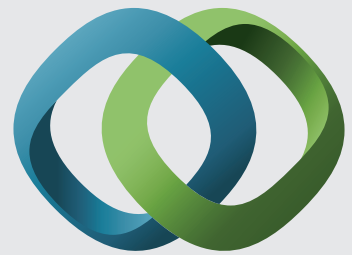

\section{Hindawi}

Submit your manuscripts at

http://www.hindawi.com
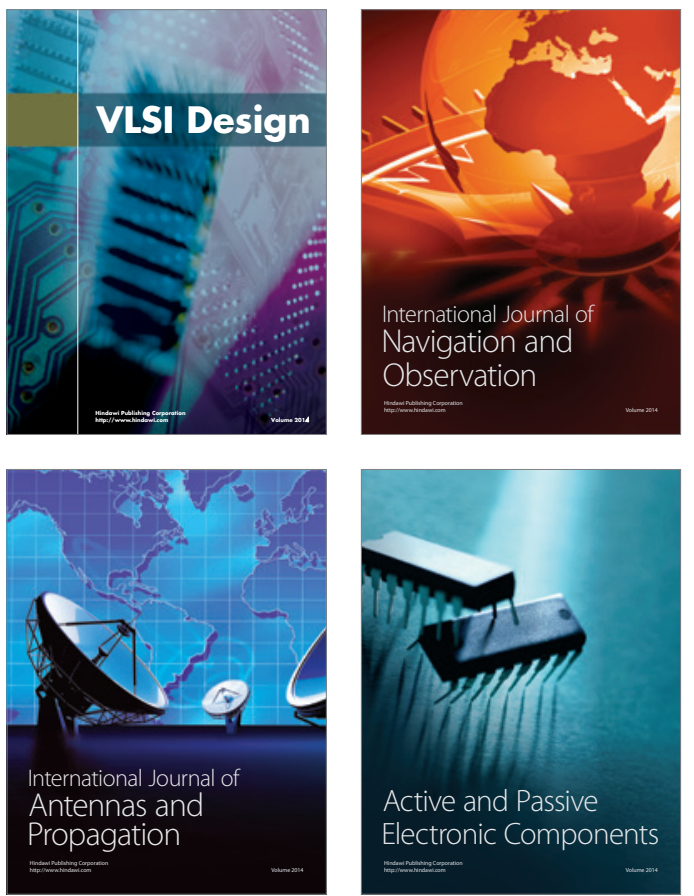
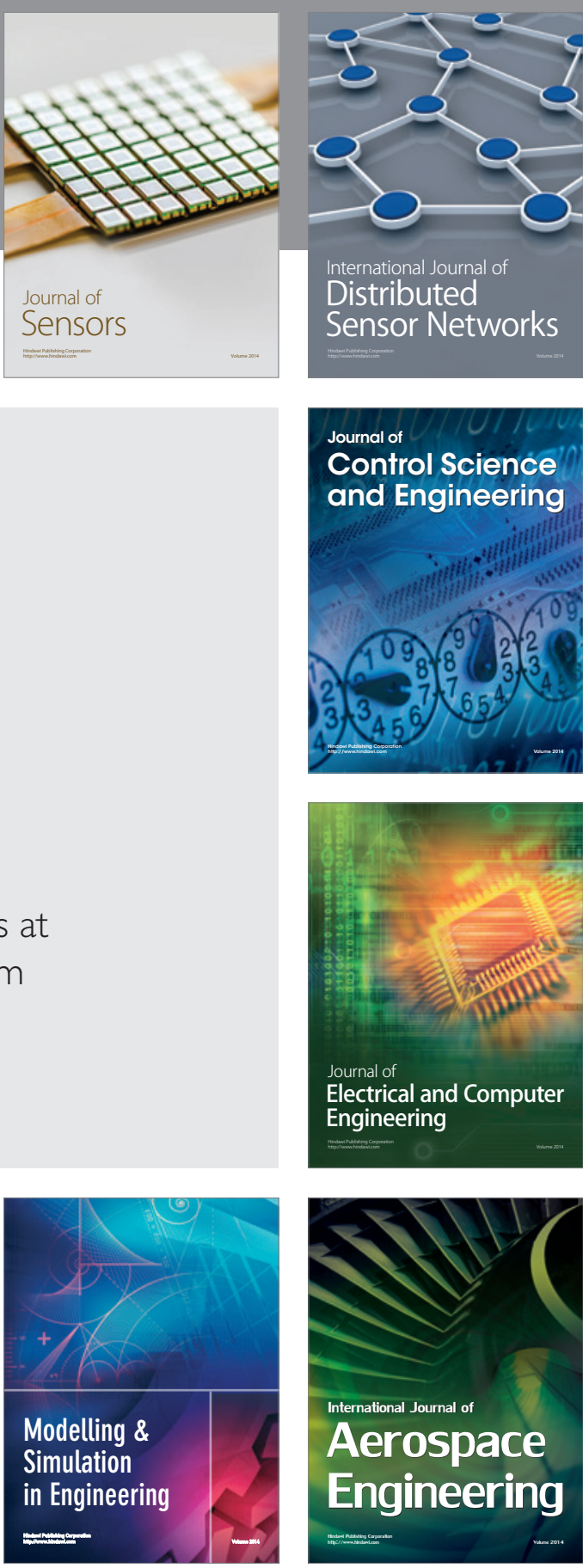

International Journal of

Distributed

Sensor Networks

Journal of

Control Science

and Engineering
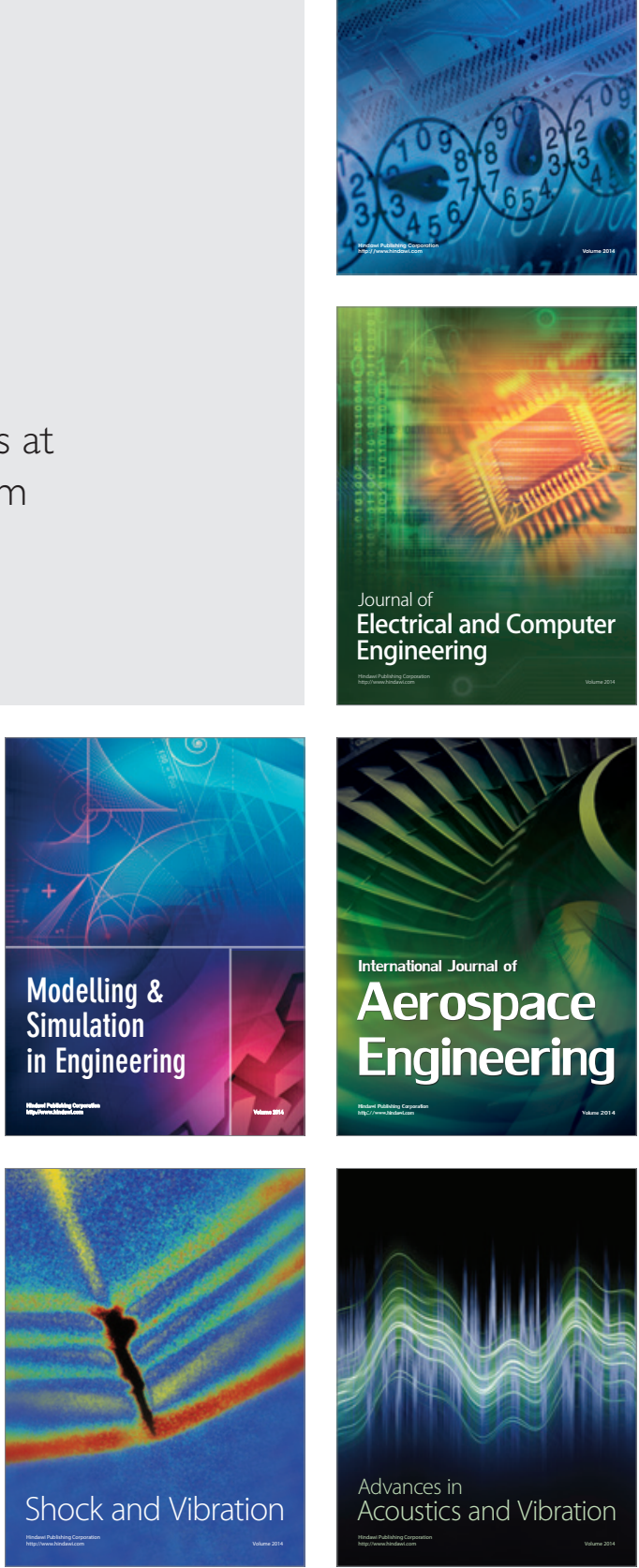